\title{
FÍSTULAS ARTERIOVENOSAS INTRA-HEPÁTICAS TRANSTUMORAIS (diagnóstico, importância e propostas de tratamento)
}

\author{
Azzo WIDMAN*, Manlio B. SPERANZINI ${ }^{* *}$, Ilka R. S. OLIVEIRA ${ }^{* * *}$, William A. SAAD ${ }^{* * * *}$, \\ Ayrton C. FRATEZZI ${ }^{* * * * *}$ e Giovanni G. CERRI ${ }^{* * * * * *}$
}

RESUMO - Achados angiográficos de quatro pacientes portadores de tumor hepático com fístula arteriovenosa transtumoral intra-hepática (duas arterioveno portais e duas arterioveno hepáticas). Concomitantemente, é mostrado o resultado após

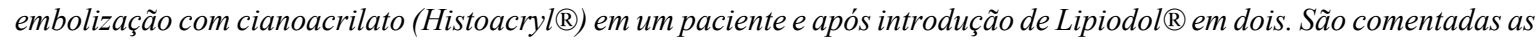
características fisiopatológicas especiais de cada um dos dois tipos de fístula e as eventuais conseqüencias da passagem da mistura quimioembolizante através do tumor: gênese de abcessos hepáticos, resposta irregular ao tratamento e complicações pulmonares. Apesar do Lipiodol $®$ atravessar a fístula arteriovenosa e se alojar nos ramos terminais da veia porta ou no pulmão, através das veias hepáticas, não há contra-indicação para o tratamento quimioembólico, mas se

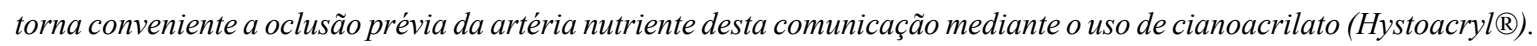
Comenta-se que, deste modo, obtém-se o alivio da hipertensão portal nas fístulas arterioveno portais e se evitam as complicações decorrentes da passagem da mistura através da comunicação arteriovenosa em ambos os tipos. O diagnóstico, apesar de dificil, é importante e habitualmente é feito por meio da angiografia abdominal; todavia a ultra-sonografia com Doppler tornada mais sensivel com o uso de contraste específico, pode alertar para a presença desta complicação vascular. Sugere-se a adoção de um algoritmo diagnóstico no estudo dos tumores hepáticos que inclua este exame, para a identificação destas fístulas, previamente ao tratamento quimioembólico. Conclui-se recomendando que este exame seja o primeiro na seqüencia diagnóstica dos tumores hepáticos, por ser procedimento não-invasivo e que a arteriografia, por ser método invasivo, seja reservada para o tratamento.

DESCRITORES - Neoplasias hepáticas. Fístula arteriovenosa. Embolização terapêutica.

Trabalho realizado no Departamento de Radiologia da Faculdade de Medicina da Universidade de São Paulo - FMUSP.

Médico Assistente Doutor da Disciplina de Cirurgia do Aparelho Digestivo da FMUSP.

** Professor Associado da Disciplina de Cirurgia do Aparelho Digestivo da FMUSP.

*** Médica Assistente Doutora da Disciplina de Radiologia da FMUSP. Chefe do Setor de Ultra-Sonografia do Instituto de Radiologia (INRAD) do Hospital das Clínicas - HC-FMUSP.

***** Professor Livre Docente da Disciplina de Cirurgia do Aparelho Digestivo da FMUSP. Professor Titular do Departamento de Cirurgia da Faculdade de Medicina da Pontifícia Universidade Católica de São Paulo, Sorocaba, SP.

****** Médico Assistente Doutor da Disciplina de Radiologia da FMUSP. Chefe de Setor de Radiologia Intervencionista do INRAD do HC-FMUSP.

******* Professor Titular do Departamento de Radiologia da FMUSP. Diretor do INRAD do HC-FMUSP

Endereço para correspondência: Dr. Azzo Widman - Rua Guilherme Moura, 302 - 05440-010 - São Paulo, SP. 


\section{INTRODUÇÃO}

As fístulas arteriovenosas adquiridas podem ter origem traumática ${ }^{(9)}$ ou decorrer de alterações teciduais por doenças que atingem o fígado e alteram a arquitetura histológica reticular do órgão, difusa ou setorialmente ${ }^{(21}$, ${ }^{24)}$. Corrobora para esta idéia a observação de que as doenças hepáticas uniformemente difusas (cirrose hepática alcoólica e virótica) ${ }^{(15)}$ e as localizadas (tumores hepáticos) apresentam comunicações fistulares arteriovenosas espontâneas ${ }^{(5)}$. Mais recentemente, uma ação terapêutica, a quimioembolização de tumores hepáticos, também tem sido incluída como causa provável deste tipo de complicação vascular ${ }^{(21)}$.

As fístulas arteriovenosas transtumorais intra-hepáticas foram descritas há muito tempo, mas devido às limitações técnicas dos meios de diagnóstico, estas complicações vasculares eram raramente identificadas ${ }^{(4,19)}$. Esta situação levou à impressão de que eram raras e, aliada à gravidade/morbidade da doença de base, fez com que não fossem suficientemente valorizadas e até mesmo ignoradas. Predominam, nesta fase, os relatos de casos isolados e que apresentavam quadros clínicos exuberantes $^{(2)}$.

A maior freqüência com que estes processos fistulares têm sido atualmente observados pode ser atribuído ao aumento dos traumas abdominais, ao uso mais freqüente de procedimentos invasivos (punção biopsia de fígado, repetição de tratamentos quimioembolizantes de tumores hepáticos ${ }^{(20)}$ ) e à indicação mais liberal dos estudos angiográficos, associada a maior sensibilidade radiográfica dos equipamentos atuais ${ }^{(21)}$. Assim, houve possibilidade de definir melhor suas características fisiopatológicas ${ }^{(8,13)} \mathrm{e}$ delinear orientações terapêuticas ${ }^{(26)}$.

O estudo angiográfico de quatro pacientes com fístula arteriovenosa intra-hepática, proporcionou-nos a oportunidade de rever as repercussões fisiopatológicas e sua importância frente aos tratamentos endovasculares dos tumores hepáticos. Outrossim, baseados nos achados radiográficos, propomos indicações para o tratamento destas complicações vasculares e um algoritmo de investigação diagnóstica.

\section{CASUÍSTICA}

Caso 1 - Paciente internada no Pronto Socorro com ascite, hematêmese e melena acompanhadas de instabilidade hemodinâmica. A ultra-sonografia identificou cirrose hepática e um tumor hepático (provável hepatocarcinoma). Após tratamento de suporte e estabilização das condições gerais, foi encaminhada para realização de estudo angiográfico com a finalidade de avaliar a extensão do tumor hepático e da hipertensão portal com vistas a possível tratamento cirúrgico.

Achado radiográfico: aspecto vascular compatível com cirrose hepática, tumor hepático no segmento III e IV, fístula arterioveno portal transtumoral de alto débito, com inversão da circulação portal (Fig. 1a, 1b). Foi realizada embolização da artéria hepática esquerda com Hystoacryl ${ }^{\circledR}+$ Lipiodol $^{\circledR}$ (1:2) (Fig. 1c). Apresentou mais

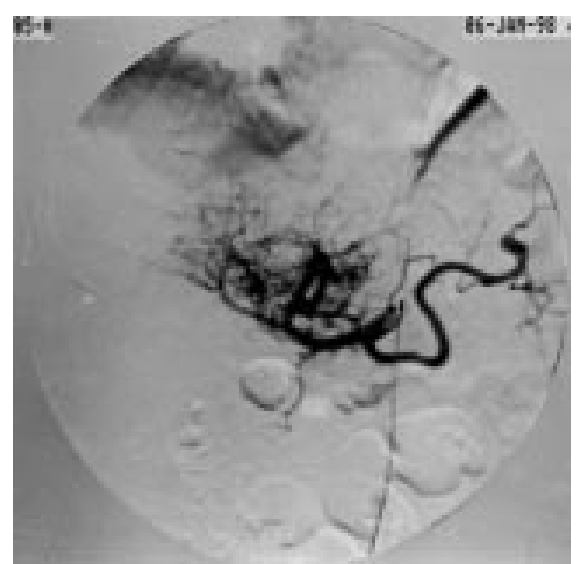

Figura 1a - Arteriografia tronco celíaco: processo tumoral no lobo esquerdo do fígado. (Tempo precoce)

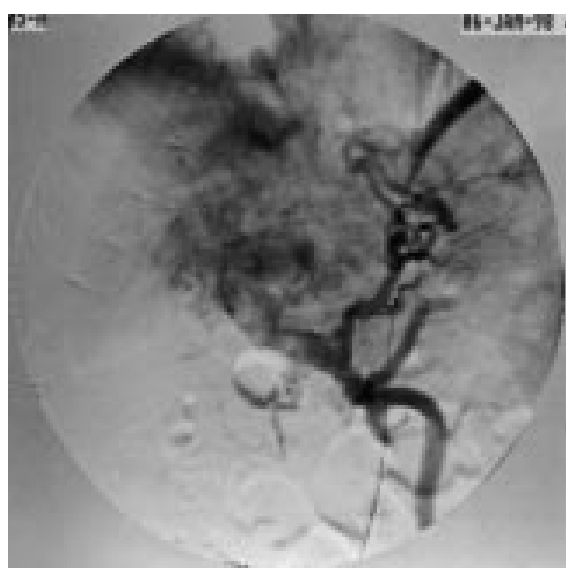

Figura 1b - Arteriografia tronco celíaco: processo tumoral no lobo esquerdo do fígado. Visualização do sistema portal, parcialmente trombosado, visualização do sistema portal com opacificação das vias de circulação hepatófuga: veia gástrica esquerda, veia esplênica, veia mesentérica superior e veia mesentérica inferior. (Tempo tardio)

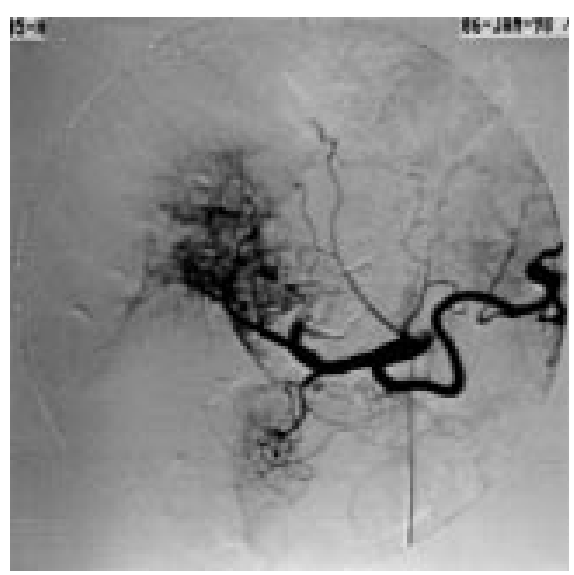

Figura 1c - Arteriografia tronco celíaco pósembolização da artéria hepática esquerda com Hystoacryl ${ }^{\circledR}$. Obstrução da artéria com desaparecimento da fístula arterioveno portal

dois episódios de melena sem repercussão hemodinâmica acentuada e teve alta hospitalar em regulares condições físicas.

Caso 2 - Paciente internada com diagnóstico de hepatocarcinoma do lobo direito do fígado. Encaminhada para avaliação angio- 
gráfica e introdução de Lipiodol ${ }^{\circledR}$. Achado angiográfico: aspecto vascular compatível com hepatocarcinoma do segmento V,VI e VII com fístula arterioveno portal transtumoral de baixo débito (Fig. 2a, 2b). Após introdução do Lipiodol ${ }^{\circledR}$, houve redução acentuada do escoamento do sangue do sistema portal do segmento afetado e acentuação da opacidade do tronco da veia porta (Fig. 2c).

Caso 3 - Paciente internado com diagnóstico de hepatocarcinoma do lobo direito do fígado. Encaminhado para avaliação angio-

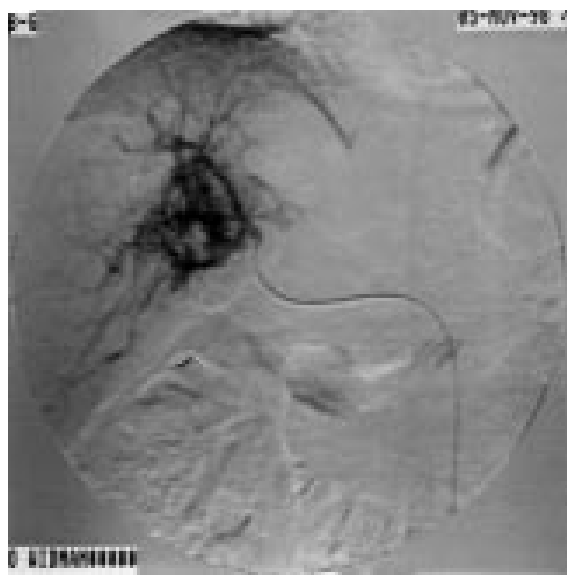

Figura 2a - Arteriografia seletiva da artéria hepática direita: presença de lesão tumoral com visualização de fístula arterioveno portal de baixo débito. (Tempo precoce)

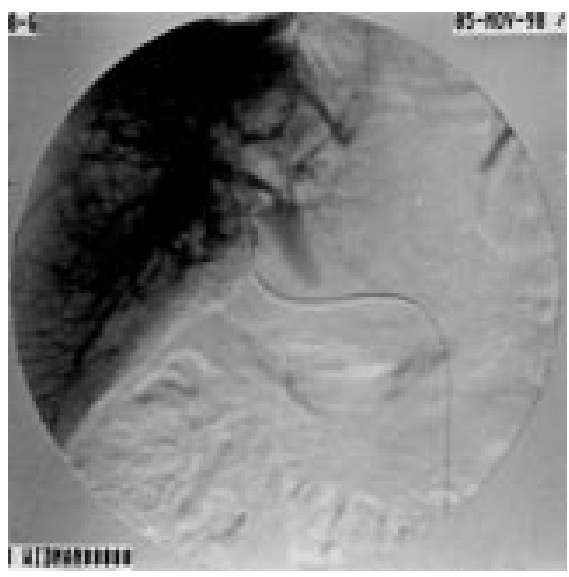

Figura 2b - Arteriografia seletiva da artéria hepática direita: presença de lesão tumoral com visualização da circulação portal. (Tempo tardio)

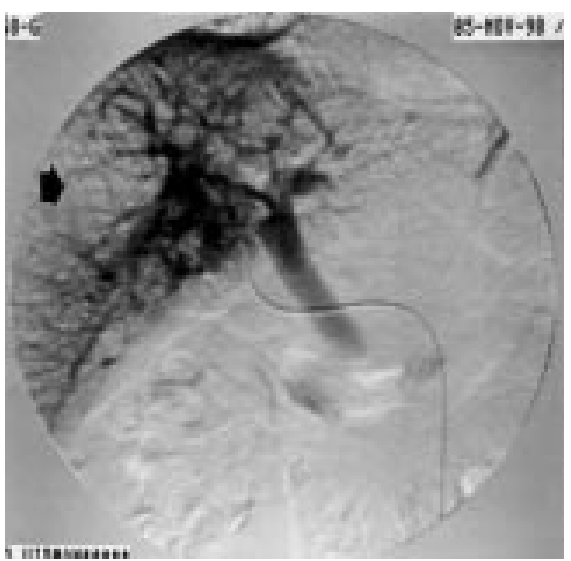

Figura 2c - Arteriografia seletiva da artéria hepática direita pós introdução de Lipiodol® (seta grossa): visualização da circulação portal. (Tempo tardio)

gráfica e introdução de Lipiodol®. Achado radiográfico: aspecto vascular compatível com hepatocarcinoma do segmento V,VI e VII com fístula arterioveno hepática transtumoral de alto débito (Fig. 3a, 3b). Após introdução do Lipiodol ${ }^{\circledR}$, houve redução acentuada do débito da fístula, porém sem desaparecimento desta (Fig. 3c).

Caso 4 - Paciente internada com recidiva de hepatocarcinoma do lobo direito do fígado. Encaminhada para avaliação angiográfica préoperatória. Achado radiográfico: variação anatômica vascular com artéria hepática direita originada da artéria mesentérica superior, presença de circulação do tipo tumoral do segmento III, IV e V dependente de ambas as fontes arteriais, presença de fístula arterioveno hepática transtumoral de baixo débito (Fig. 4a, 4b). Paciente foi operada e o tumor ressecado.

\section{DISCUSSÃO}

\section{Fisiopatologia}

As fístulas arteriovenosas transtumorais intrahepáticas podem ser agrupadas, conforme sua origem em: arterioveno portais (casos 1 e 2) e em arterioveno hepáticas

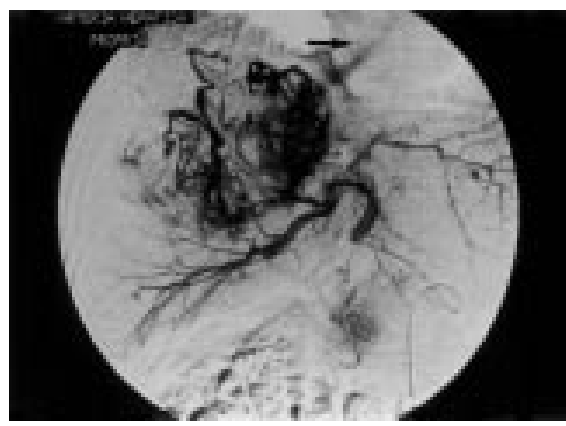

Figura 3a - Arteriografia seletiva da artéria hepática: lesão tumoral na parte central do fígado. Visualização da drenagem venosa precoce através de fístula arterioveno hepática (seta)

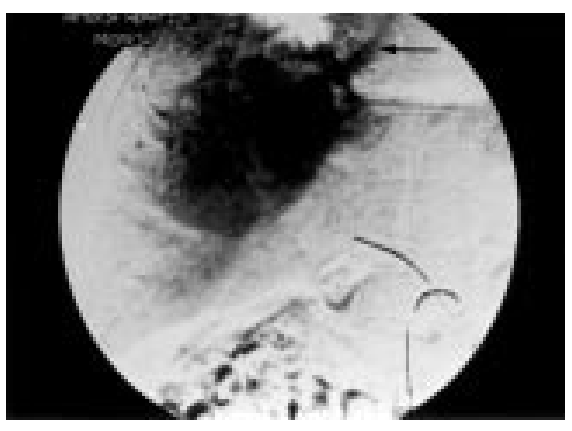

Figura 3b - Arteriografia seletiva da artéria hepática: lesão tumoral na porção central do fígado. Drenagem sangüínea por veia hepática (seta). (Tempo tardio)

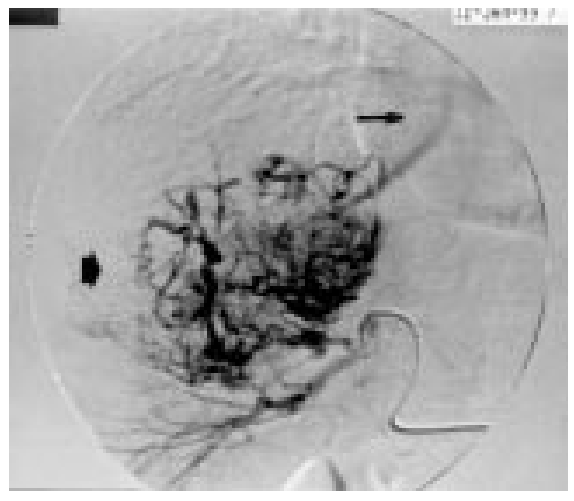

Figura 3c - Arteriografia seletiva da artéria hepática pós-introdução de Lipiodol®: retenção do Lipiodol® (seta grossa) e diminuição do fluxo através da fístula arterioveno hepática (seta) 


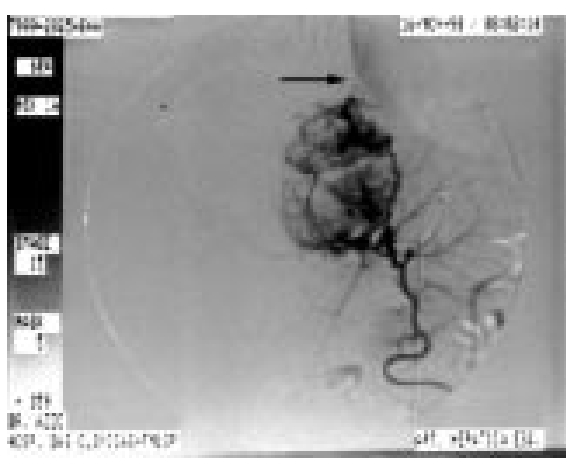

Figura 4a - Arteriografia do tronco celíaco: variação anatômica. Opacificação de lesão tumoral na porção central do fígado

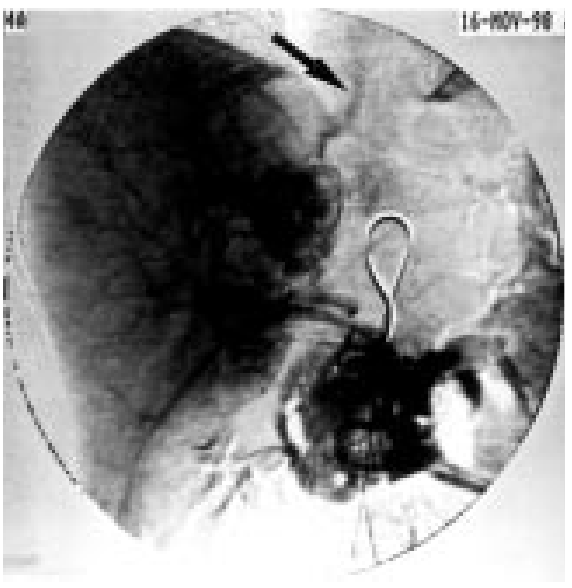

Figura 4b - Arteriografia seletiva da artéria hepática direita pela artéria mesentérica superior: lesão tumoral na porção média do figado

( $\operatorname{casos} 3$ e 4). Por obedecer sempre às limitações do binômio magnitude da fonte arterial e capacidade de acomodação do setor venoso comprometido, uma gama quase infinita de aspectos, que dependem do fluxo, pode ser observada nestas comunicações vasculares (Fig. 1b, 2b, 3b, 4a, 4b). O volume de sangue que passa através destas fístulas depende do diâmetro dos vasos arteriais que se comunicam diretamente com o sistema venoso de drenagem local através do tumor. Esta situação é modulada pela capacidade dos vasos do sistema esplâncnico e do cardiopulmonar que, habitualmente, tendem a disfarçar a real dimensão destas comuni- cações arteriovenosas. Da interação destes dois fatores, volume de sangue e capacidade de acomodação do sistema venoso, é que surgem os sintomas e sinais que, quando presentes, possibilitam a identificação deste tipo de fístula, mas quando ausentes tornam sua presença dificilmente dignosticável.

Aspectos fisiopatológicos distintos podem ser notados nos dois tipos:

- as fistulas arterioveno portais transtumorais (comunicação entre um ramo arterial do tumor hepático e a veia porta), adicionam componente pressorial considerável ao sistema portal, contribuindo na gênese de ascites rebeldes e sangramentos digestivos de difícil tratamento. Por acontecerem habitualmente em fígados com cirrose, tendem a exacerbar a hipertensão portal subjacente (Fig. 1b).

- as fístulas arterioveno hepáticas transtumorais (comunicação entre um ramo arterial do tumor hepático e uma veia de drenagem do mesmo do setor do fígado), só em condições especiais produzem sintomas e/ou sinais que possam sugerir sua presença. Esta situação se deve à capacidade de tamponamento pela rede capilar pulmonar e pela reserva funcional cardíaca.

\section{Importância dos processos fistulares frente ao tratamento quimioembólico dos tumores hepáticos}

A introdução intra-arterial intra-hepática de Lipiodol ${ }^{\circledR}$ em pacientes com fístula arterioveno portal (caso 2) ou arterioveno hepática (caso 3 ), mostrou em ambas as situações, que este agente embolizante, apesar de ficar retido no parênquima hepático (seta grossa), não tem a capacidade de ocluir esta comunicação vascular indepen- dentemente do débito desta (Fig. 2b, 3c seta fina).

Quando um tumor hepático com fístula arterioveno portal é tratado com uma mistura de quimioterápicos e agentes oclusivos (Lipiodol ${ }^{\circledR}$ e/ou partículas: amido, Ivalon ${ }^{\circledR}$ ou Gelfoam $^{\mathbb{R}}$ ), parte deste material passa para o sistema venoso diretamente através da fístula e se aloja nos ramos terminais da veia porta do segmento hepático tratado (Fig. 2c). Assim, além da obstrução arterial provocada nos ramos arteriais terminais intratumorais, haverá a oclusão de um conjunto de ramos portais relacionados com a comunicação arteriovenosa proporcionalmente ao débito da fístula (Fig. 2c).

Nestas condições, em virtude da oclusão concomitante arterial e portal, o resultado será a isquemia mais acentuada do setor tratado no fígado, comprometendo, ao mesmo tempo, o tumor (irrigado, preferencialmente, pela vertente arterial) e o tecido hepático (irrigado, preferencialmente, pela vertente portal). A vantagem de uma isquemia tão ampla é a garantia de que está sendo alcançado o desiderato da proposta terapêutica que é de tornar o tumor amplamente isquêmico pela oclusão de todas as suas fontes de irrigação (arterial e portal). No entanto, esta desvascularização do segmento hepático tratado, pode provocar também necrose de tecido hepático normal circunjacente ao tumor e, assim, facilitar a ocorrência de abscesso neste local ${ }^{(21)}$. Neste sentido também, a necrose do parênquima hepático, que nestes casos está reduzido em virtude da cirrose subjacente, pode levar à insuficiência hepática transitória ou definitiva, comprometendo o resultado do tratamento ${ }^{(7,20)}$.

A importância de uma fístula arterioveno hepática transtumoral para o tratamento quimioembólico de tumores hepáticos, está no fato de que uma parte variável da mistura quimioembolizante (quimioterápicos + Lipiodol ${ }^{\mathbb{B}} \mathrm{e} /$ ou agente particulado) pode atravessar a comunicação arteriovenosa (Fig. 3c). 
Neste caso, os agentes embolizantes (partículas e Lipiodol ${ }^{\circledR}$ ) se alojarão na rede capilar pulmonar podendo causar abcessos ${ }^{(22)} \mathrm{e}$ vários graus de embolia que, em condições extremas, pode concorrer para o óbito do paciente ${ }^{(7,25)}$. A parte líquida da mistura que passou pela comunicação, se distribuirá para todo o organismo, sem agir com eficiência no tumor hepático.

Deste modo, a quimioterapia sistêmica e embolização pulmonar (indesejáveis), resultantes desta passagem da mistura quimioembolizante através da fístula, serão proporcionais ao débito desta comunicação arteriovenosa e evitarão a ação físico-farmacológica local no tumor hepático (desejável). Esta situação talvez possa explicar resultados irregulares do tratamento quimioembólico e até mesmo a sua falha, pois somente uma parte reduzida da mistura quimioembolizante ficará retida no tumor.

\section{Técnicas de tratamento das fístulas arteriovenosas transtumorais hepáticas}

A embolização da artéria hepática esquerda com Hystoacryl $®$ eliminou o fator de exacerbação da hipertensão portal subjacente na paciente (caso 1) com fístula arterioveno portal transtumoral de grande débito (Fig. 1c) e apresentando hemorragia digestiva de difícil tratamento.

A generalização da prática da quimioembolização dos tumores hepáticos, devido aos benefícios que este tratamento tem proporcionado, associada à freqüência com que estas comunicações arteriovenosas têm sido diagnosticadas, torna impraticável a simples contraindicação do método quando estas fístulas são identificadas ${ }^{(21)}$. Esta conduta foi reavaliada e estas complicações vasculares passaram a ter uma orientação terapêutica específica ${ }^{(18,26)}$.

Assim, no caso de uma fístula arterioveno portal transtumoral (Fig. 1b), com quadro clínico exuberante, o tratamento visando sua oclusão se torna prioritário e a quimioembolização do tumor deve ser postergada. Nesta situação, após avaliar arteriograficamente o paciente, sugerimos tratar, de início, apenas a fístula para que os sintomas e sinais da hipertensão portal (hemorragia digestiva alta de difícil controle e ascite rebelde), exacerbados pelo aumento considerável da pressão venosa no território esplâncnico regridam (Fig. 1c). O tratamento deve ser aplicado também nos casos em que a fístula tenha baixo fluxo, com vistas a impedir a necrose desnecessariamente ampla de tecido hepático, apesar da rede portal estar suportando o aumento do contingente circulatório sem complicações clínicas (Fig. 2b).

Esta meta pode ser atingida mediante a oclusão, unicamente, da artéria nutriente do segmento do tumor relacionado à fístula. Vários agentes têm sido utilizados para tratamento de fístulas arteriovenosas intrahepáticas de origem traumática (molas, balões oclusores). Seus resultados têm sido irregulares, pois ocluem a artéria longe do foco fistular possibilitando que ramos arteriais colaterais intra-hepáticos, através de comunicações intraparenquimatosas, restabeleçam a circulação ${ }^{(6)}$. O cianoacrilato (Hystoacryl ${ }^{\circledR}$ ), raramente utilizado para embolização de tumores hepáticos ${ }^{(1,12)}$, foi empregado também, uma vez, para tratamento de fístula arterioveno portal ${ }^{(10)}$. A velocidade de polimerização desta substância pode ser alterada mediante a adição dosada de Lipiodol $^{\circledR(1,26)}$, de modo a permitir que se oclua a artéria de forma mais abrangente (Fig. 1c) e mais próximo da região da comunicação arteriovenosa $^{(9,12)}$, impedindo o recrutamento da circulação colateral.

Nos casos em que há uma fístula arterioveno hepática transtumoral, as complicações pulmonares serão diretamente proporcionais ao débito desta anastomose vascular (Fig. 3b, 4a, 4b). Da mesma forma, a falha parcial ou total do tratamento quimioembólico do tumor hepático pode ser atribuída à passagem direta do material quimioembolizante através do tumor.

A dificuldade de se identificar este tipo de fístula ${ }^{(14)}$ torna o dano circulatório pulmonar o principal marcador na fase que se segue imediatamente após o tratamento. Assim, naquelas condições em que o débito da fístula é acentuado e/ou um grande volume de Lipiodol ${ }^{\circledR}$ é injetado, a lesão pulmonar pode se tornar exuberante e até provocar o óbito do paciente ${ }^{(17)}$. Todavia, este dano pode não ser percebido em virtude da grande extensão da rede capilar pulmonar total comparada ao volume do material particulado e Lipiodol ${ }^{\circledR}$ que a atinge, o que, no entanto, não diminui a importância do prejuízo.

Nas fístulas arterioveno hepáticas, da mesma forma que nas fístulas arterioveno portais, sugerimos tratar a artéria nutriente da comunicação vascular e portanto a lesão fistular, antes de se iniciar o tratamento quimioembolizante do tumor hepático.

\section{Proposta de algoritmo para investigação diagnóstica dos tumores hepáticos}

Do exposto, verificamos que, antes do procedimento de quimioembolização de tumores hepáticos, o diagnóstico das fístulas arteriovenosas transtumorais é importante para que se possam evitar complicações.

A angiografia abdominal tem sido o método de imagem mais freqüentemente utilizado para o diagnóstico das fístulas $\operatorname{arteriovenosas}^{(1,3,5,8,13,19,21)}$; todavia, quando realizada durante os procedimentos iniciais da quimioembolização, nem sempre identifica claramente estas comunicações.

O examinador, nesta fase, atento à disposição anatômica das artérias do tronco celíaco e à perviedade do sistema mesentérico ou 
esplenoportal, pode não perceber a presença de uma fístula arteriovenosa transtumoral intrahepática. Isto se dá, principalmente, quando não há sinais clínicos que alertem para sua presença (fístula arterioveno hepática) (Fig. 3b, 4a, 4b) e/ou quando ela é de baixo débito com sinais radiográficos discretos (fístula arterioveno portal) (Fig. 2b). Deste modo, o mascaramento da fístula resulta na passagem direta do material quimioembolizante através do tumor, levando às conseqüências já descritas.
Atualmente, a ultra-sonografia com Doppler tem oferecido subsídios importantes para a identificação de fistulas vasculares intra-hepáticas e eventualmente tem sido utilizada para este fim ${ }^{(4,15)}$. Esta metodologia identifica as comunicações através do mapeamento colorido (padrão em mosaico) e pelas alterações espectrais (turbulência de fluxo) observadas na região da fístula ${ }^{(10,11,23)}$. Possivelmente esta técnica não-invasiva, realizada sistematicamente nos pacientes com tumor hepático, forneça indícios seguros da presença de ambas comunicações vasculares (fístulas arterioveno portais e/ou arterioveno hepáticas).

A inclusão desta técnica diagnóstica, a ser realizada em primeiro lugar no algoritmo da investigação dos tumores hepáticos, permite que sejam tomadas as precauções necessárias e as medidas terapêuticas adequadas para a correção desta complicação vascular e tornar mais seguro e eficaz o tratamento quimioembolizante dos tumores hepáticos.

Widman A, Speranzini MB, Oliveira IRS, Saad WA, Fratezzi AC, Cerri GG. Intrahepatic transtumoral arteriovenous fistulae (diagnosis, importance and therapeutic proposals). Arq Gastroenterol 2000;37(1):13-9.

ABSTRACT - The authors present the angiographic aspects of four patients having an arteriovenous trashepatic transtumoral fistula (two arterioportal and two arteriovenous). The angiographic results of the occlusion with cyanoacrylate (Hystoacryl $\left.{ }^{\circledR}\right)$ of the right hepatic

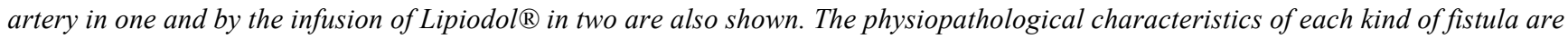
discussed and the consequences of the passage of the quimioembolizing mixture through the tumor, regarding intrahepatic abcess formation, irregular clinical results and pulmonary complications are commented. These complications make the diagnosis of a transtumoral transhepatic arteriovenous fistula, previuosly to the chemoembolic treatment important, even though beeing frequently difficult, because of the reduced caliber of the feeding artery and eventual low transfistular bloodflow. The efficient occlusion of the arterioportal fistula with Hystoacryl ${ }^{\circledR}$ was favourably compared to the infusion of Lipiodol $\AA$, wich was unable to occlude the arteriovenous fistula. The contraindication to perform chemoembolic treatment of hepatic tumors, when an intrahepatic transtumoral arteriovenous fistula is present and the embolization of the fistular feeding atery is stressed. Ultrasonography using color Doppler and sono-enhancing contrast is appointed as the ideal noninvasive means of making the diagnosis of an hepatic transtumoral fistula and makes it eligible to be the first test in the algorithm for the appraisal of hepatic tumors.

HEADINGS - Liver neoplasms. Arteriovenous fistula. Embolization, therapeutic.

\section{REFERÊNCIAS BIBLIOGRÁFICAS}

1. Bartolozzi C, Lencioni R, Ricci P, Paolicchi A, Rossi P, Passariello R. Hepatocellular carcinoma treatment with percutaneous ethanol injection: evaluation with contrast-enhanced color Doppler US. Radiology 1998;209:387-93.

2. Berghammer P, Pfeffel F, Winkelbauer F, Wiltschke C, Schenk T, Lammer J, Müller C, Zielinski C. Arterial hepatic embolization of unresectable hepatocellular carcinoma using a cyanoacrylate/ Lipiodol mixture. Cardiovasc Intervent Radiol 1998;21:214-8.
3. Chung JW, Park JH, Im J-G, Han JK, Han MC. Pulmonary oil embolism after transcatheter oily chemoembolization of hepatocellular carcinoma. Radiology 1993;187:689-93.

4. Chung JW, Park JH, Han JK, Choi BI, Han CH, Lee H-S, Kim CY. Hepatic tumors: predisposing factors for complications of transcatheter oily chemoembolization. Radiology 1996;198: $33-40$.

5. Chung JW. Transcatheter arterial chemoembolization of hepatocellular carcinoma. Hepatogastroenterology 1998;45 Suppl:1236-41. 
6. Cubiella J, Sans M, Llovet JM, Bustamente J, Ferrer A, Caballeria J, Rodés J. Pulmonary abscess as a complication of transarterial embolization of multinodular hepatocellular carcinoma. Am J Gastroenterol 1997;92:1942-3.

7. Grattagliano A, Rappacini GL, Comaldo G, Pompili M, Marino P, Mastromatteo AM, Cotroneo AR, Gasbarrini G. Spontaneous intrahepatic portosystemic venous shunt in patient with cirrhosis: diagnosis by combined color Doppler and pulsed Doppler ultrasonography. Liver 1977;17:307-10.

8. Groupe d'Étude et de Traitement du Carcinome Hépatocellulaire. A comparison of lipiodol chemoembolization and conservative treatment for unresectable hepatocellular carcinoma. N Engl J Med 1995;332:1256-61.

9. Itzchak Y, Adar R, Bogokowski H, Mozes M, Deutch V. Intrahepatic arterial portal communications: angiographic study. AJR Am J Roentgenol 1974;121:384-7.

10. Kerlan KK, Bank WO, Hoddick WK, Pogany AC, Sollenberger $\mathrm{RD}$. Occlusion of a hepatic arterio-portal vein fistula with bucrylate. Cardiovasc Intervent Radiol 1983;6:138-40.

11. Kim AY, Choi BI, Kim TK, Han JK, Yun EJ, Lee KY, Han MC: Hepatocellular carcinoma: power Doppler US with a contrast agent - Preliminary results. Radiology 1998;209:135-40.

12. Kozuka S, Sasa R, Kakumu S. An enormous intrahepatic shunt between portal vein and hepatic one. Angiology 1975;26:365-71.

13. Kudo M, Tomita S, Tochio H, Minowa K, Todo A. Intrahepatic portosystemic venous shunt: diagnosis by color Doppler imaging. Am J Gastronterol 1993;88:723-9.

14. Morse SS, Sniderman KW, Rapoport S, Ross GR, Glickman MG. Hepatoma, arterioportal shunting and hyperkinetic portal hypertension: therapeutic embolization. Radiology $1985 ; 155: 77-82$.

15. Nagasue N, Inokuchi K, Kobayashi M, Saku M. Hepatoportal arteriovenous fistula in primary carcinoma of the liver. Surg Gynecol Obstet 1977;145:504-08.

16. Ngan H. Interventional radiology for hepatocellular carcinoma. Dig Surg 1995;12:65-71.
17. Ngan H, Peh WCG. Arteriovenous shunting in hepatocellular carcinoma: its prevalence and clinical significance. Clin Radiol 1997;52:36-40.

18. Okuda K, Musha H, Yamasaki T, Jinnouchi S, Nagasaki Y, Kubo Y, Shimokawa Y, Nakayama T, Kojiro M, Sakamoto 2, Nakashima T. Angiographic demonstration of intrahepatic arterio-portal anastomoses in hepatocellular carcinoma. Radiology $1977 ; 122: 53-8$.

19. Park JH, Cha SH, Han JK, Han MC. Intrahepatic portosystemic venous shunt. AJR Am J Roentgenol 1990;155:527-8.

20. Puglionisi A, Giovani V, Snider F, Camilli S, Cina G. A propos d'un cas de fistule artério-veineuse hépatico-portale: problèmes de physiopathologie et clinique et revue de la littérature. J Chir (Paris) 1980;117:607-19.

21. Sakamoto I, Aso N, Nagaoki K, Matsuoka Y, Uetani M, Ashizawa K, Iwanaga S, Mori M, Morikawa M, Fukuda T, Hayashi K, Matsunaga N. Complications associated with transcatheter arterial embolization for hepatic tumors. Radiographics 1998;18:605-19.

22. VanWay CW-III, Crane JM, Riddell DH, Foster JH. Arteriovenous fistula in the portal circulation. Surgery 1977;70:876-90.

23. Vauthey J-N, Reinhard JT, Helmberger T, Gertsch P, Forsmark C, Caridi J, Reed A, Langham MR, Lauwers GY, Goffette P, Lerut J. The arterioportal fistula syndrome: clinicopathologic features, diagnosis and therapy. Gastroenterology 1997;113:1390-401.

24. Widman A, Speranzini MB, Oliveira IK, Fratezi AC, Cerri GG. Portal hypertension with arterioportal fistula through an hepatic tumor (diagnosis and treatment). [In Press 1999].

25. Winkelbauer FW, Niederle B, Pietschmann F, Thurner S, Wilding R, Prokesch R, Lammer J. Hepatic artery embolotherapy of hepatic metastases from carcinoid tumors: Value of using a mixture of cyanoacrylate and ethiodized oil. AJR Am J Roentgenol 1995; 165:323-7.

26. Woodcock J. The potential of echo-enhancement. Adv Echoenhancement 1996;1:2-11.

Recebido para publicação em 5/3/1999. Aprovado para publicação em 21/6/1999. 